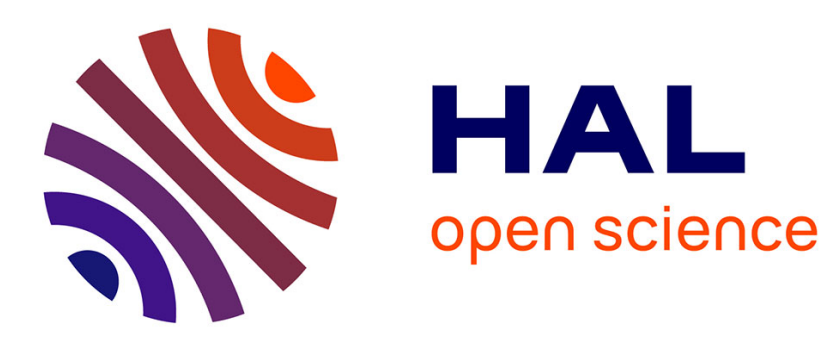

\title{
On the nonlinear destabilization of stably stratified shear flow
}

Nadia Mkhinini, Thomas Dubos, Philippe Drobinski

\section{To cite this version:}

Nadia Mkhinini, Thomas Dubos, Philippe Drobinski. On the nonlinear destabilization of stably stratified shear flow. Journal of Fluid Mechanics, 2013, 731 (september), pp.443-460. 10.1017/jfm.2013.374 . hal-01099073

\section{HAL Id: hal-01099073 https://hal.science/hal-01099073}

Submitted on 1 Jan 2015

HAL is a multi-disciplinary open access archive for the deposit and dissemination of scientific research documents, whether they are published or not. The documents may come from teaching and research institutions in France or abroad, or from public or private research centers.
L'archive ouverte pluridisciplinaire HAL, est destinée au dépôt et à la diffusion de documents scientifiques de niveau recherche, publiés ou non, émanant des établissements d'enseignement et de recherche français ou étrangers, des laboratoires publics ou privés. 


\title{
On the nonlinear destabilization of stably stratified shear flow
}

\author{
Nadia Mkhinini, Thomas Dubos $\dagger$ and Philippe Drobinski \\ Laboratoire de Météorologie Dynamique/IPSL, École Polytechnique, 91128 Palaiseau, France
}

(Received 25 August 2012; revised 28 June 2013; accepted 15 July 2013;

first published online 15 August 2013)

A weakly nonlinear analysis of the bifurcation of the stratified Ekman boundary-layer flow near a critical bulk Richardson number is conducted and compared to a similar analysis of a continuously stratified parallel shear flow subject to Kelvin-Helmholtz instability. Previous work based on asymptotic expansions and predicting supercritical bifurcation at Prandtl number $\operatorname{Pr}<1$ and subcritical bifurcation at $\operatorname{Pr}>1$ for the parallel base flow is confirmed numerically and through fully nonlinear temporal simulations. When applied to the non-parallel Ekman flow, weakly nonlinear analysis and fully nonlinear calculations confirm that the nature of the bifurcation is dominantly controlled by $\operatorname{Pr}$, although a sharp threshold at $\operatorname{Pr}=1$ is not found. In both flows the underlying physical mechanism is that the mean flow adjusts so as to induce a viscous (respectively diffusive) flux of momentum (respectively buoyancy) that balances the vertical flux induced by the developing instability, leading to a weakening of the mean shear and mean stratification. The competition between the former nonlinear feedback, which tends to be stabilizing, and the latter, which is destabilizing and strongly amplified as $\operatorname{Pr}$ increases, determines the supercritical or subcritical character of the bifurcation. That essentially the same competition is at play in both the parallel shear flow and the Ekman flow suggests that the underlying mechanism is valid for complex, non-parallel stratified shear flows.

Key words: bifurcation, nonlinear dynamical systems, stratified flows

\section{Introduction}

Shear and stratification compete to respectively destabilize and stabilize many flows, especially environmental flows. This competition is captured by the Miles-Howard criterion, establishing the critical value of the local Richardson number beyond which an inviscid stratified shear flow is linearly stable (Howard 1961; Miles 1961). This criterion describes well the onset of the primary instability of many flows, especially continuously stratified parallel shear flows (Klaassen \& Peltier 1985), and the more complicated stratified Ekman boundary-layer flow, which forms near boundaries in rotating flows and presents both similarities and qualitative differences with the parallel shear flow (Brown 1972). However this criterion does not address nonlinear effects arising as the primary instability develops. The nonlinear development of Kelvin-Helmholtz billows has been investigated for Richardson numbers far away from its critical value (Cortesi, Yadigaroglu \& Banerjee 1998; Staquet 2009). Here we 
are interested in characterizing weakly nonlinear effects arising when the Richardson number is close to its critical value.

Lilly (1966) identified two branches in the linear stability analysis of the Ekman flow, one associated with a viscous instability and another due to an inflectionpoint instability, also referred to as type I or cross-flow instability. The role of these instabilities in the transition to turbulence of more complex, non-stratified flows, especially the flow over rotating $\operatorname{disc}(\mathrm{s})$, has been extensively investigated (Lingwood 1997; Davies \& Carpenter 2003; Pier 2003; Viaud, Serre \& Chomaz 2008). In the presence of stratification and at a sufficiently high Reynolds number, the inflection-point branch is stabilized according to the Miles-Howard criterion: although the critical bulk Richardson number is quite small, the local Richardson number computed at the first inflection point is close to the theoretical value of $1 / 4$ (Brown 1972). Until the recent work by Mkhinini, Dubos \& Drobinski (2013), the nonlinear development of the instability had been studied only in the fully turbulent regime (Coleman, Ferziger \& Spalart 1992) or without stratification (Iooss, Nielsen \& True 1978; Haeusser \& Leibovich 2003; Dubos, Barthlott \& Drobinski 2008). In the nonstratified case, weakly nonlinear analysis shows that the bifurcation occurring at the lowest Reynolds number, which corresponds to the viscous instability, is supercritical (Iooss et al. 1978; Haeusser \& Leibovich 2003). Dubos et al. (2008) computed fully nonlinear equilibrated flows resulting from the saturation of the inflection-point branch and found that this bifurcation is supercritical too. Mkhinini et al. (2013) extended this work to the stratified case by increasing the bulk Richardson number $R i$ until the critical value $R i=R i_{c}$ was reached. For Prandtl number $\operatorname{Pr}=1$ the deviation of the equilibrated flow from the basic Ekman profile vanished at $R i=R i_{c}$, indicating a supercritical bifurcation, while at $\operatorname{Pr}=4$ the deviation of the equilibrated flow from the basic Ekman profile had a finite amplitude at $R i=R i_{c}$, indicating a subcritical bifurcation.

Churilov \& Shukhman (1987), extending and correcting Brown, Rosen \& Maslowe (1981), investigated nonlinear feedbacks in a continuously stratified parallel shear flow with a linear profile for buoyancy and a tanh profile for velocity. In what follows we refer to this basic flow as the Kelvin-Helmholtz $(\mathrm{KH})$ profile. Churilov \& Shukhman (1987) is based on a weakly nonlinear theory, and matched asymptotic expansions in the limit of large Reynolds number $R e \rightarrow \infty$, yielding the dominant contribution to the first Landau coefficient. Their main prediction, confirmed by temporal direct numerical simulations (Lott \& Teitelbaum 1992) is that the net nonlinear feedback is stabilizing if the Prandtl number $\operatorname{Pr}<1$ and destabilizing if $\operatorname{Pr}>1$. This control of nonlinear feedbacks by $\operatorname{Pr}$ can be understood qualitatively as the result of a competition between the nonlinear weakening of the mean shear and mean stratification, although the interaction between the unstable wave and its second harmonic also contributes.

Given the similarities and also differences between the $\mathrm{KH}$ and Ekman flows, this work investigates whether the mechanism found to dominate the nonlinear feedbacks in the $\mathrm{KH}$ flow is of sufficiently wide validity to also apply to the stably stratified Ekman flow. In $\S 2$ we present the base flows and the most important quantities involved in the weakly nonlinear analysis. In $\S 3$ the salient conclusions arrived at by the asymptotic theory of Churilov \& Shukhman (1987) are verified numerically. In $\S 4$ we analyse the development of perturbations added to the stratified Ekman flow near the bifurcation $R i=R i_{c}$. A partial exploration of the parameter space indicates that the Prandtl number $P r$, rather than the Reynolds number or the wave vector of the instability, is indeed the primary factor on which the nature of the bifurcation depends. We relate this dependence to the modifications of the mean flow induced by the 
nonlinear development of the unstable wave, as with the KH flow. Nevertheless, not all contributions to the first Landau coefficient have the same behaviour as a function of $R e$ and $P r$ in both flows, the most robust mechanism being the amplification as $P r$ increases of the destabilizing feedback due to the change in mean stratification. Our results are summarized and discussed in $\S 5$. Details of the weakly nonlinear theory are provided in appendix A and the numerics are described in appendix B.

\section{Basic flows and notation}

\subsection{Basic flows}

The flows we consider obey the Boussinesq equations:

$$
\begin{gathered}
\nabla \cdot \boldsymbol{U}=0, \quad \frac{\partial \boldsymbol{U}}{\partial t}+\boldsymbol{U} \cdot \boldsymbol{\nabla} \boldsymbol{U}+\frac{1}{R o} \boldsymbol{e}_{z} \times \boldsymbol{U}+\nabla P=\frac{1}{R e} \Delta \boldsymbol{U}+B \sqrt{R i} \boldsymbol{e}_{z}, \\
\frac{\partial B}{\partial t}+\boldsymbol{U} \cdot \nabla B=\frac{1}{\operatorname{RePr}} \Delta B-w \sqrt{R i},
\end{gathered}
$$

where $\boldsymbol{U}$ is the velocity, $B$ the buoyancy, $P$ is the pressure, $w$ is the vertical velocity and $e_{z}$ is the unit vector in the $z$ direction. In the non-dimensional equations (2.1)-(2.2), velocities have been scaled by a velocity scale $U$, lengths by a spatial scale $\delta$, time by the advective scale $\delta / U$ and buoyancy by $U N$ where $N$ is the Brunt-Väisälä frequency of the background linear stratification. The flow is controlled globally by the bulk Richardson number $R i=N^{2} /(U / \delta)^{2}$, the Reynolds number $R e=U \delta / v$, the Rossby number $R o=U / f \delta$ and the Prandtl number $\operatorname{Pr}=\nu / \kappa$, where $v$ is the kinematic velocity, $\kappa$ is the kinematic diffusivity of buoyancy and $f$ the Coriolis parameter.

The stratified free shear basic flow that we consider is:

$$
U_{K H}=\tanh z, \quad V_{K H}=0, \quad W_{K H}=0, \quad B_{K H}=z \sqrt{R i}
$$

in a non-rotating frame, i.e. $1 / R o=0$ in (2.1). In what follows, we refer to this basic flow as the Kelvin-Helmholtz profile. The velocity scale is the maximum velocity and the length scale is given by the shear thickness.

The Ekman profile, given by:

$$
U_{E}=1-\mathrm{e}^{-z} \cos z, \quad V_{E}=\mathrm{e}^{-z} \sin z, \quad W_{E}=0, \quad B_{E}=z \sqrt{R i},
$$

is an exact solution of the Boussinesq equations (2.1)-(2.2) for the steady three-way balance between the pressure gradient, Coriolis and frictional forces, which specifies the characteristic length scale $\delta=\sqrt{2 v / f}$, hence $R o=R e / 2$ (Ekman 1905). The velocity scale is defined as the geostrophic velocity, to which the flow velocity tends far away from the solid boundary present at $z=0$.

\subsection{Amplitude equation}

We shall analyse the nonlinear development of infinitesimal perturbations $(\boldsymbol{u}, b)$ added to either the $\mathrm{KH}$ flow or the stratified Ekman flow near the bifurcation $R i=R i_{c}$. For this we derive an amplitude equation obeyed by the small amplitude $A$ of perturbations:

$$
\frac{\mathrm{d} A}{\mathrm{~d} t}=\left(\sigma_{1}+\mu|A|^{2}\right) A, \quad \frac{1}{2} \frac{\mathrm{d}}{\mathrm{d} t}|A|^{2}=\left(\operatorname{Re}\left(\sigma_{1}\right)+\operatorname{Re}(\mu)|A|^{2}\right)|A|^{2}
$$

where $\sigma_{1}$ is the growth rate of the linear instability, $\mu$ is the first Landau coefficient and $\operatorname{Re}$ denotes the real part. The perturbations themselves are expanded in powers 
of $A$, starting with the normal mode of the shear instability. In order to define $\mu$ unambiguously, the normal mode is scaled to unit total energy $T E=K E+P E$, with $K E=\int \overline{\boldsymbol{u}}^{2} \mathrm{~d} z$ the kinetic energy and $P E=\int \overline{b^{2}} \mathrm{~d} z$ potential energy, where the overbar denotes horizontal averaging. The normal mode is sinusoidal in the $x, y$ directions and defines the fundamental harmonic. New harmonics appear as higher powers of $A$ are considered (see appendix A for a full derivation).

Notice that (2.3) is considered here as steady (frozen), which means that we neglect the viscous diffusion of the basic KH profile. Indeed (2.3) is a steady solution only of the inviscid Boussinesq equations. This seems problematic as the growth rates we consider will typically be small since $R i \simeq R i_{c}$. However, as discussed in detail by Churilov \& Shukhman (1987), there exists a range of amplitudes for which the weakly nonlinear theory is valid and at the same time the amplitude evolves much faster than the time scale of diffusion of the base flow, which can therefore be consistently neglected.

As derived in appendix A, we obtain $\mu$ for any value of $\operatorname{Pr}, \operatorname{Re}, \boldsymbol{k}$ where $\boldsymbol{k}$ is the wave vector of the eigenmode. For each $\operatorname{Pr}, \operatorname{Re}, \boldsymbol{k}$, there is an $\operatorname{Ri}(\operatorname{Pr}, \operatorname{Re}, \boldsymbol{k})$ such that $\sigma_{1}<0$ for $R i>R i_{c}$. We also define $\boldsymbol{k}_{c}(\operatorname{Pr}, \operatorname{Re})$ such that $R i_{c}\left(\operatorname{Pr}, \operatorname{Re}, \boldsymbol{k}_{c}\right)=$ $\max _{k}\left(R i_{c}(\operatorname{Pr}, R e, k)\right)$. The value of $\mu$ is computed at $R i \leqslant R i_{c}(R e, \operatorname{Pr}, \boldsymbol{k})$ from a compatibility condition (A 20) involving the adjoint eigenmode and nonlinear source terms induced by the development of the instability. At $R i=R i_{c}$, where $\operatorname{Re}\left(\sigma_{1}\right)=0$, the sign of $\operatorname{Re}(\mu)$ determines the stability of the laminar equilibrium $A=0$. If $\operatorname{Re}(\mu)<0$ the nonlinear effects are stabilizing and limit the growth of the amplitude while if $\operatorname{Re}(\mu)>0$, the nonlinear effects are destabilizing and amplify the growth of the roll amplitude. In the latter case a finite-amplitude equilibrium or no equilibrium at all exists at the bifurcation depending on higher-order terms not included in (2.5).

A detailed expression for $\mu$ is shown in appendix A. We decompose $\mu$ as:

$$
\mu=\mu^{[0]}+\mu^{[2]}
$$

where the contribution $\mu^{[0]}$ results from a modification of the mean flow and the contribution $\mu^{[2]}$ is due to the second harmonic. The main question we address here is whether $\mu$ is positive or negative, depending on what control parameters, and according to which dynamical process. To know which contribution is the main cause of the sign change of $\mu$, we also decompose $\mu^{[0]}$ as:

$$
\mu^{[0]}=\mu_{\boldsymbol{u}}^{[0]}+\mu_{b}^{[0]} .
$$

Here $\mu_{b}^{[0]}$ is the contribution due to the modification of the mean buoyancy and $\mu_{u}^{[0]}$ is the contribution due to the modification of the mean velocity.

\section{Nonlinear feedbacks in the Kelvin-Helmholtz flow}

\subsection{The asymptotic theory of Churilov \& Shukhman (1987)}

Churilov \& Shukhman (1987) derive an asymptotic value for the first Landau coefficient $\mu$ as $R e \rightarrow \infty$ at fixed $\operatorname{Pr}$ and $R i=R i_{c}\left(R e, \operatorname{Pr}, \boldsymbol{k}_{c}\right)$. Note that the Landau coefficient $\mu$ and the various contributions of $\mu$ are real due to the mirror symmetry of the flow. Their asymptotic theory relies on a multiple-time-scale expansion and matched asymptotics. A viscous critical layer develops near the inflection point $z=0$, which requires careful asymptotic expansion in the critical layer and matching between the outer expansion and the expansion in the critical layer. Salient results obtained by (Churilov \& Shukhman 1987) are that: 


\begin{tabular}{lccccccccccc}
\hline $\operatorname{Pr}$ & $R i_{c}$ & $k_{x}$ & $k_{y}$ & $\mu$ & $\mu^{[2]}$ & $\mu^{[0]}$ & $\mu_{\boldsymbol{u}}^{[0]}$ & $\mu_{b}^{[0]}$ & $\mu_{b x}^{[0]}$ & $\mu_{b z}^{[0]}$ & $|A|_{e q}^{2}$ \\
0.25 & 0.2465 & 0.70 & 0 & $-\mathbf{1 9 . 3}$ & -2.8 & -16.5 & -9.2 & -7.3 & -11.8 & $\mathbf{4 . 4}$ & $9.1 \times 10^{-5}$ \\
0.7 & 0.2465 & 0.70 & 0 & $-\mathbf{7 . 0}$ & -0.7 & -6.3 & -5.5 & -0.8 & -12.8 & $\mathbf{1 2 . 0}$ & $2.5 \times 10^{-4}$ \\
1 & 0.2465 & 0.70 & 0 & $\mathbf{0}$ & 0.6 & -0.6 & -4.7 & 4.1 & -13.2 & $\mathbf{1 7 . 3}$ & 0.045 \\
4 & 0.2465 & 0.70 & 0 & $\mathbf{7 0 . 0}$ & 11.4 & 58.6 & -2.8 & 61.4 & -13.8 & $\mathbf{7 5 . 0}$ & -
\end{tabular}

TABLE 1. Values of the Landau coefficient $\mu$ and its various contributions $\mu^{[2]}, \mu^{[0]}, \mu_{\boldsymbol{u}}^{[0]}, \mu_{b}^{[0]}, \mu_{b x}^{[0]}$ and $\mu_{b z}^{[0]}$ for $\mathrm{KH}$ base flow at $R e=1000$. The equilibrium amplitude $|A|_{e q}$ is computed at $R i=0.99 R i_{c}$ using (3.4).

(a) $\mu^{[0]}$ and $\mu^{[2]}$ both change sign precisely at $\operatorname{Pr}=1$, with $\mu<0$ for $\operatorname{Pr}<1$ and $\mu>0$ for $\operatorname{Pr}>1$;

(b) the thickness of the critical layer scales like $R e^{-1 / 3}$;

(c) both contributions $\mu^{[0]}$ and $\mu^{[2]}$ scale like $R e$ but the mean-flow contribution $\mu^{[0]}$ dominates.

Furthermore the change of sign of $\mu$ at $\operatorname{Pr}=1$ can be explained qualitatively as follows: the variation of the local Richardson number which governs the flow stability is determined by a competition between the nonlinear reduction of the mean shear and that of mean stratification. The former is a stabilizing effect, while the latter is destabilizing and enhanced by a larger $\operatorname{Pr}$. Therefore for sufficiently large $\operatorname{Pr}$ the destabilizing effect dominates and $\mu>0$.

\subsection{Numerical verification of the asymptotic theory}

To provide a quantitative comparison between the several contributions of $\mu$, we give their values computed at $\operatorname{Re}=1000, \operatorname{Pr}=0.25,0.7,1,4$ in table 1 . We find that $\mu_{u}^{[0]}$ is always negative so modification of the mean shear is always stabilizing. Therefore we now focus on the contribution of the mean buoyancy which can be further decomposed as:

$$
\mu_{b}^{[0]}=\mu_{b x}^{[0]}+\mu_{b z}^{[0]}
$$

where $\mu_{b x}^{[0]}$ depends on horizontal gradients of the linear eigenmode while $\mu_{b z}^{[0]}$ depends on the modification of the horizontal-mean buoyancy gradient (see appendix $\mathrm{A}$ ); $\mu_{b z}^{[0]}$ depends strongly on $\mathrm{Pr}$ while $\mu_{b x}^{[0]}$ depends weakly on the Prandtl number. Overall, the change in sign of $\mu$ is dominantly due to the strong amplification of $\mu_{b z}^{[0]}$ as $\operatorname{Pr}$ is increased, with a smaller contribution from $\mu^{[2]}$ which also increases with Pr. Because the destabilizing feedback $\mu_{b z}^{[0]}$ competes with the stabilizing feedbacks $\mu_{u}^{[0]}, \mu_{b x}^{[0]}$ which depend weakly on $\mathrm{Pr}$, this amplification leads to a change of the net feedback from stabilizing at $\operatorname{Pr}=0.7$ to destabilizing at $P r=4$. On the other hand, the various contributions cancel each other at $\operatorname{Pr}=1$ leading to $\mu \simeq 0$. This means that the change in sign occurs precisely at $\operatorname{Pr}=1$, in agreement with Churilov \& Shukhman (1987).

In figure $1(a)$, we examine the mean shear modification $\mathrm{d} u_{2}^{[0]} / \mathrm{d} z$ and mean stratification modification $\mathrm{d} b_{2}^{[0]} / \mathrm{d} z$ at a fixed $R e(R e=1000)$. The interpretation of these profiles is that the mean velocity and buoyancy profiles change as the perturbation grows according to:

$$
\boldsymbol{u}^{[0]}=\boldsymbol{U}_{K H}(z)+|A|^{2} \boldsymbol{u}_{2}^{[0]}(z), \quad b^{[0]}=B_{K H}(z)+|A|^{2} b_{2}^{[0]}(z)
$$


(a)

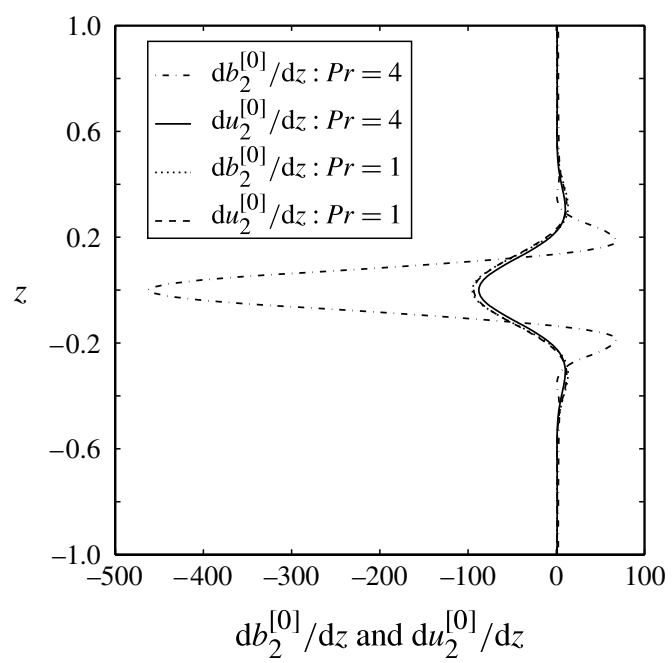

(b)

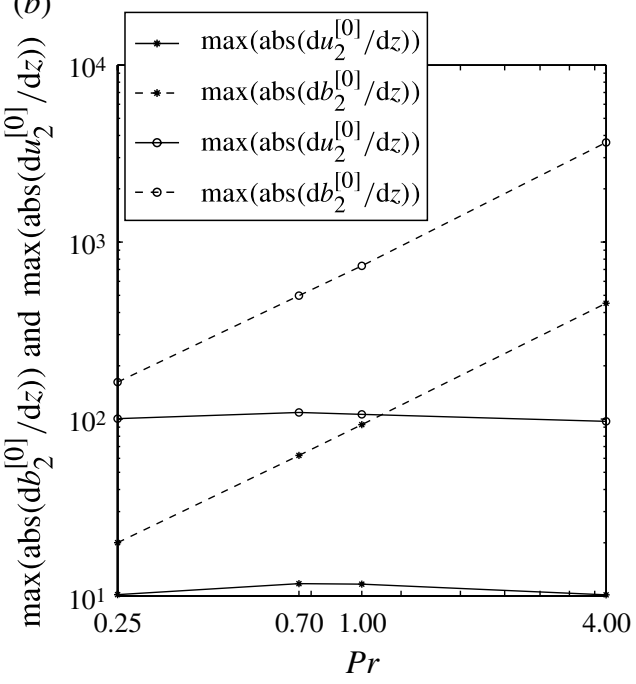

Figure 1. (a) Nonlinear modification of mean shear $\mathrm{d} u_{2}^{[0]} / \mathrm{d} z$ and mean stratification $\mathrm{d} b_{2}^{[0]} / \mathrm{d} z$ at $\operatorname{Pr}=1$ and $\operatorname{Pr}=4, \operatorname{Re}=1000$. (b) $\max \left(\left|\mathrm{d} u_{2}^{[0]} / \mathrm{d} z\right|\right)$ and $\max \left(\left|\mathrm{d} b_{2}^{[0]} / \mathrm{d} z\right|\right)$ as a function of $\operatorname{Pr}$ at $R e=10000$ (circles) and $R e=1000$ (dots) for KH flow.

where $\boldsymbol{u}^{[0]}$ is the horizontal-mean velocity and $b^{[0]}$ is the horizontal-mean buoyancy. They correspond to a weakening of the mean shear and mean stratification, the latter being amplified at higher $P r$. The dependence of $b_{2}^{[0]}$ and $u_{2}^{[0]}$ on the Prandtl number can be analysed quantitatively as follows. The mean flow modification is determined by (A 12), which simplifies at $R i=R i_{c}$ for the mean flow since $\sigma_{1}+\bar{\sigma}_{1}=0$. Using the expression (A 6) for the linearized operator acting on the mean flow, (A 12) reduces to:

$$
\frac{1}{\operatorname{Re}} \frac{\mathrm{d} u_{2}^{[0]}}{\mathrm{d} z}=u_{1}^{[1]} \overline{w_{1}^{[1]}}+\text { c.c., } \quad \frac{1}{\operatorname{Re} \operatorname{Pr}} \frac{\mathrm{d} b_{2}^{[0]}}{\mathrm{d} z}=b_{1}^{[1]} \overline{w_{1}^{[1]}}+\text { c.c. },
$$

i.e. the mean flow adjusts so as to induce a viscous (respectively diffusive) flux of momentum (respectively buoyancy) that balances the vertical flux induced by the developing instability. For fixed $R e, \mathrm{~d} u_{2}^{[0]} / \mathrm{d} z$ can vary as $P r$ increases only if the right-hand side (r.h.s.) of (3.3) does, while $\mathrm{d} b_{2}^{[0]} / \mathrm{d} z$ can vary both due to changes in the r.h.s. and due to an increase of $P r$, even at fixed r.h.s. We plot in figure $2(a)$ the velocity field $u_{1}$ and the buoyancy field $b_{1}$ of the eigenmode for two different $\operatorname{Pr}, R e=1000$ and $R i \simeq R i_{c}$ The velocity eigenmode $u_{1}$ depends very little on $\operatorname{Pr}$. Thus the r.h.s of (3.3) determining $u_{2}^{[0]}$ and $\mu_{u}^{[0]}$ is also almost constant as $\operatorname{Pr}$ varies. On the other hand the buoyancy eigenmode $b_{1}$ becomes more vertically confined as $\mathrm{Pr}$ is increased, and also more intense. To verify how the profiles $\mathrm{d} u_{2}^{[0]} / \mathrm{d} z$ and $\mathrm{d} b_{2}^{[0]} / \mathrm{d} z$ scale with $\operatorname{Pr}$, it is sufficient to consider the maxima $\max \left(\left|\mathrm{d} u_{2}^{[0]} / \mathrm{d} z\right|\right)$ and $\max \left(\left|\mathrm{d} b_{2}^{[0]} / \mathrm{d} z\right|\right)$, shown in figure $1(b)$ as a function of $\operatorname{Pr}$ at fixed $\operatorname{Re}(\operatorname{Re}=1000$ and $\operatorname{Re}=10000)$. The dependence of $\mathrm{d} b_{2}^{[0]} / \mathrm{d} z$ on $P r$ is not affected when we increase the Reynolds number. Combined with the proportionality of $\mathrm{d} b_{2}^{[0]} / \mathrm{d} z$ to $\operatorname{Pr}$, these dependences lead to the rapid growth of $\mu_{b z}^{[0]}$ with $\operatorname{Pr}$ observed in table 1 .

We now check that the Landau coefficient $\mu$ computed numerically as described in appendix A behaves as predicted by Churilov \& Shukhman (1987) in the 

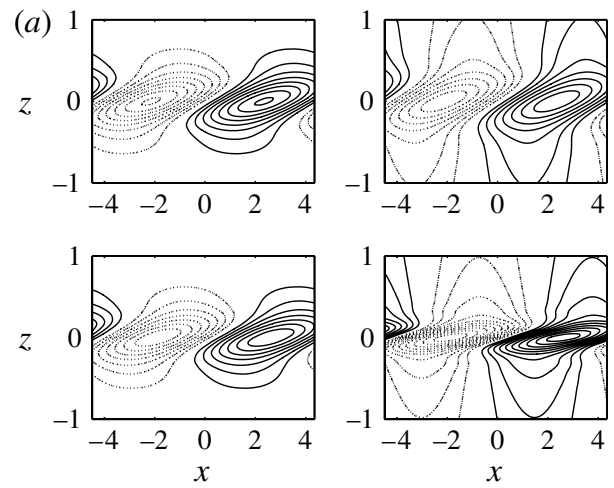

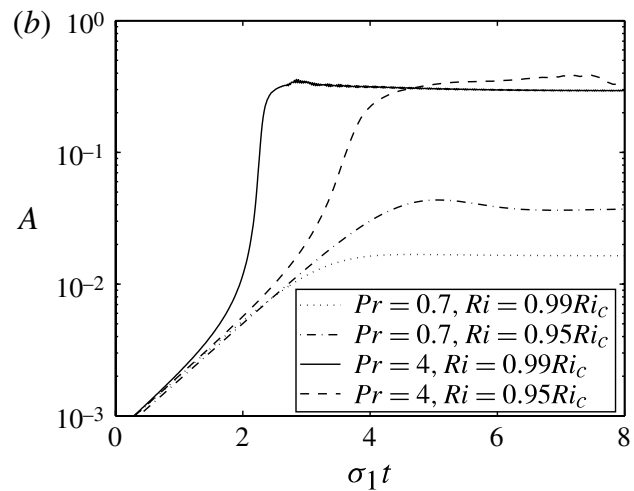

FIGURE 2. (a) KH eigenmodes $u_{1}(x, z)=u_{1}^{[1]}(z) \exp (\mathrm{i} \boldsymbol{k} \cdot \boldsymbol{x})+$ c.c. (left column) and $b_{1}(x, z)=b_{1}^{[1]}(z) \exp (\mathrm{i} \boldsymbol{k} \cdot \boldsymbol{x})+$ c.c. (right column) for $\operatorname{Pr}=1$ (top row) and $\operatorname{Pr}=4$ (bottom row), $R e=1000, R i \simeq R i_{c}$. Contour level interval is 0.2 and negative contours are dotted. (b) Nonlinear saturation of $\mathrm{KH}$ instability near $R i=R i_{c}$ at $R e=1000$ and $\operatorname{Pr}=0.7$ and $\operatorname{Pr}=4$.
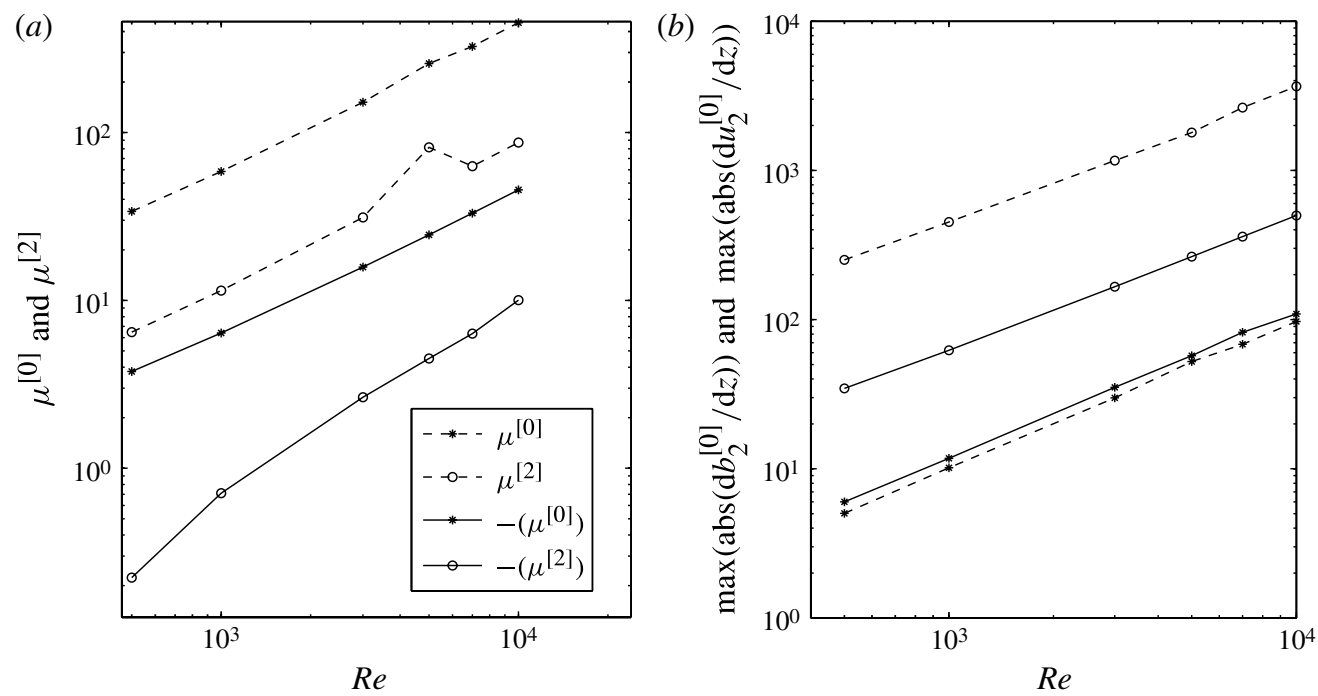

FIgURE 3. (a) $\mu^{[0]}$ (stars) and $\mu^{[2]}$ (circles) as a function of $\operatorname{Re}$ for $\mathrm{KH}$ flow. At $\operatorname{Pr}=4$ (dashed line), $\mu^{[0]}$ and $\mu^{[2]}$ are positive while at $P r=0.7$ (solid line), $\mu^{[0]}$ and $\mu^{[2]}$ are negative and we plot $-\mu^{[0]}$ and $-\mu^{[2]}$. (b) $\max \left(\operatorname{abs}\left(\mathrm{d} u_{2}^{[0]} / \mathrm{d} z\right)\right)(\operatorname{dots})$ and $\max \left(\operatorname{abs}\left(\mathrm{d} b_{2}^{[0]} / \mathrm{d} z\right)\right)$ (circles) as a function of $R e$.

range $500 \leqslant R e \leqslant 10000$ and $0.25 \leqslant P r \leqslant 4$. We first verify at fixed $\operatorname{Pr}$ that $\mu^{[0]}$ and $\mu^{[2]}$ scale like $R e$ and that the mean-flow contribution $\mu^{[0]}$ dominates. For that we plot $\mu^{[0]}$ and $\mu^{[2]}$ as a function of $\operatorname{Re}$ (figure $3 a$ ) for $\operatorname{Pr}=0.7$ and $\operatorname{Pr}=4$. This figure shows that both $\mu^{[0]}$ and $\mu^{[2]}$ scale as $\operatorname{Re}$ and also that for $\operatorname{Pr}>4, \mu^{[0]} \sim 5 \mu^{[2]}$.

Next we plot $\max \left(\mathrm{d} u_{2}^{[0]} / \mathrm{d} z\right)$ and $\max \left(\mathrm{d} b_{2}^{[0]} / \mathrm{d} z\right.$ ) (figure $\left.3 b\right)$ as a function of $R e$ at a fixed $P r$, and find that they scale with $\operatorname{Re}$ consistently with (3.3) and with Churilov \& Shukhman (1987). 


\subsection{Numerical verification of the weakly nonlinear theory}

To verify the validity of the weakly nonlinear theory for the $\mathrm{KH}$ profile, finally we perform fully nonlinear two-dimensional simulations of stratified $\mathrm{KH}$ flow at $R i$ slightly below $R i_{c}$ at $P r=0.7$ and $P r=4$. We compute numerically $R i_{c}(R e=1000, P r=0.7)=0.2465$, with the corresponding wavenumber $k_{c}=0.70$. Four different simulations with $P r=0.7,4$ and $R i=0.95 R i_{c}, 0.99 R i_{c}$ are initialized with $(\boldsymbol{u}, b)=\left(\boldsymbol{U}-\boldsymbol{U}_{K H}, B-B_{K H}\right)=A\left(\boldsymbol{u}_{1}, b_{1}\right)$ where $A=10^{-4}$ and $\left(\boldsymbol{u}_{1}, b_{1}\right)$ is the linear eigenmode computed for each $(P r, R i)$ pair at $k=k_{0}$, normalized to unit total energy $\int \overline{\boldsymbol{u}_{1}^{2}+b_{1}^{2}} \mathrm{~d} z=1$ where the overbar denotes horizontal averaging. Due to this normalization $E(t)=\int \overline{\boldsymbol{u}^{2}+b^{2}} \mathrm{~d} z=|A|^{2}+O\left(|A|^{4}\right)$ for small $A$, so that $A$ can be approximated by $E^{1 / 2}$ for the purposes of this subsection. Figure $2(b)$ presents the amplitude $E^{1 / 2} \simeq|A|$ of the nonlinear perturbation as a function of time rescaled by the growth rate of the linear instability $\sigma_{1}=\sigma_{1}\left(R e=1000, \operatorname{Ri}, \operatorname{Pr}, k=k_{c}\right)$. By design all four curves collapse during the linear phase $E^{1 / 2} \sim \exp \left(\sigma_{1} t\right)$. Later, deviations from the linear behaviour $E^{1 / 2} \sim \exp \left(\sigma_{1} t\right)$ confirm that the nonlinearities are stabilizing at $\operatorname{Pr}=0.7\left(E^{1 / 2}<\exp \left(\sigma_{1} t\right)\right)$ and destabilizing at $\operatorname{Pr}=4\left(E^{1 / 2}>\exp \left(\sigma_{1} t\right)\right.$ before saturation).

When $\mu<0$ the equilibrium amplitude $|A|_{e q}$ reached by $E^{1 / 2}$ as $t \rightarrow \infty$ can be computed from $\mu$ and the linear growth rate $\sigma_{1}$ as

$$
|A|_{e q}^{2}=-\sigma_{1} / \mu \simeq \frac{R i_{c}-R i}{\mu} \frac{\mathrm{d} \sigma_{1}}{\mathrm{~d} R i} .
$$

At $\operatorname{Pr}=0.7, R i=0.99 R i_{c}$ (3.4) is verified quite well with $|A|_{e q}^{2}=2.7 \times 10^{-4},-\sigma_{1} / \mu=$ $1.8 \times 10^{-3} / 7=2.5 \times 10^{-4}$ (table 1 , rightmost column). Furthermore $\left|A_{e q}\right|$ decreases roughly like $\sqrt{R i_{c}-R i}$ as $R i$ approaches $R i_{c}$ (the ratio of saturated amplitudes at $R i=0.95 R i_{c}$ compared to $R i=0.99 R i_{c}$ is $\sim 2 \simeq \sqrt{0.05 / 0.01}$ ) while the amplitude at saturation remains the same for $\operatorname{Pr}=4$. This confirms directly that the weakly nonlinear expansion is quantitatively accurate and that the bifurcation of the $\mathrm{KH}$ flow is supercritical at sufficiently low $P r$ and subcritical at sufficiently high $P r$.

We have also used these nonlinear simulations to estimate directly the Landau coefficient from a linear least-squares fit of the relationship between the total energy $E$ and its growth rate $E^{-1} \mathrm{~d} E / \mathrm{d} t$. In fact $E=|A|^{2}+\alpha|A|^{4}+O\left(|A|^{6}\right)$ yields $E^{-1} \mathrm{~d} E / \mathrm{d} t=2 \sigma_{1}+2\left(\mu+\alpha \sigma_{1}\right) E^{2}+O\left(E^{3}\right) \simeq 2 \sigma_{1}+2 \mu E^{2}$ for near-critical $R i$. This estimate agrees within 5-10\% with the Landau coefficient computed from the weakly nonlinear analysis.

\section{Nonlinear feedbacks in the stratified Ekman layer}

In $\S 3$ we verified that the Prandtl number predominantly controls the supercritical/subcritical nature of the bifurcation of the continuously stratified $\mathrm{KH}$ flow near $R i=R i_{c}$ as predicted in Churilov \& Shukhman (1987). The weakly nonlinear analysis suggests that the underlying physical mechanism is the competition between a Prandtl-number-independent weakening of the mean shear and a Prandtl-numberdependent weakening of the mean stratification as the instability develops. This mechanism does not seem to depend on the details of the basic flow. To find out whether this nonlinear destabilization mechanism is indeed valid for a more general stratified shear flow, we repeat here our weakly nonlinear analysis on the Ekman flow which is affected by rotation and the presence of a boundary and is not unidirectional. 

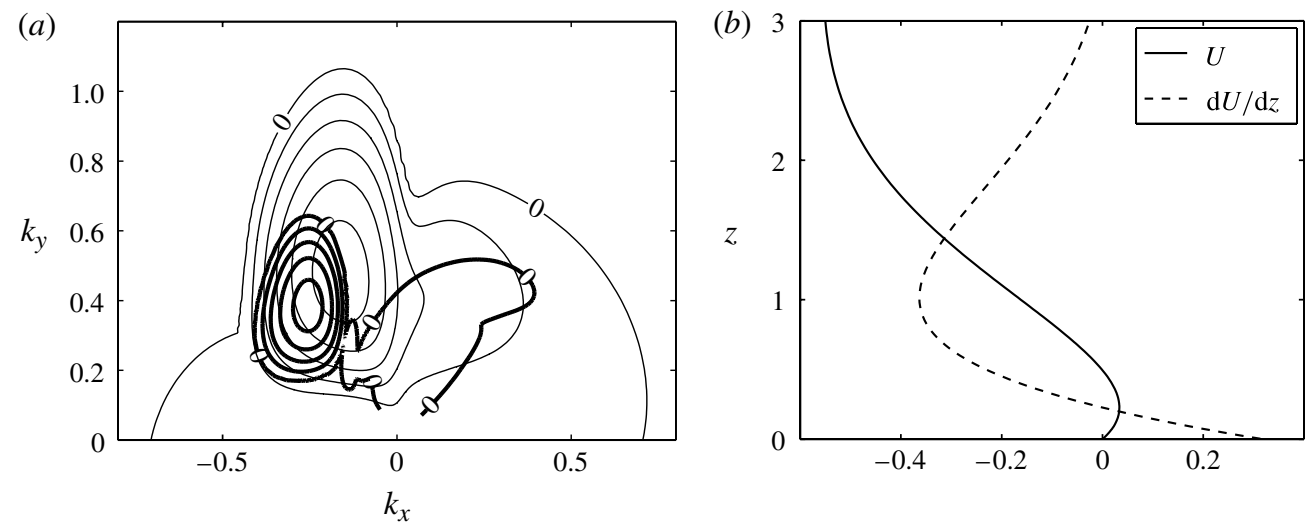

FIGURE 4. (a) Real part of the growth rate $\sigma_{1}(R e=1000, R i, \operatorname{Pr}, \boldsymbol{k})$ of the primary instability as a function of the wave vector $\boldsymbol{k}$, at $R i=0$ (thin, contour interval 0.005 ) and $R i=0.015, P r=4$ (thick, contour interval 0.002). (b) Ekman flow velocity along wave vector $\boldsymbol{k}$ where $\boldsymbol{k}$ corresponds to the fastest-growing mode.

Infinitesimal perturbations with horizontal wave vector $\boldsymbol{k}=\left(k_{x}, k_{y}\right)$ added to the Ekman flow grow exponentially in time if $R i<R i_{c}(R e, \operatorname{Pr}, \boldsymbol{k})$ where $R i_{c}$ is the critical bulk Richardson number above which the linear instability is suppressed. The overall critical value $R i_{c}(R e, P r)=\max _{k} R i_{c}(R e, P r, k)$ depends somewhat on the Reynolds number $R e$ but very little on the Prandtl number (Mkhinini et al. 2013). We display in figure $4(a)$ the real part of the growth rate $\sigma_{1}(R e=1000, R i=0, \boldsymbol{k})$ of the primary instability. The non-trivial dependence of $\operatorname{Re}\left(\sigma_{1}\right)$ on $\boldsymbol{k}$ is due to the veering in the Ekman profile. In what follows we focus on the region of wave vector space $\left(k_{x}, k_{y}\right) \in[-0.5,0.1] \times[0,1.2]$ where $\sigma_{1}$ is close to its maximum value, which corresponds to the inflection-point instability.

\subsection{Parameters controlling the nature of the bifurcation}

We now explore the parameter space to determine if $\operatorname{Re}(\mu)$ depends on $\operatorname{Pr}$ independently of $R e$ and $\boldsymbol{k}$. Note that the basic flow is no longer symmetric, hence the Landau coefficient and its different contributions are complex numbers.

We plot in figure 5 the real part of the Landau coefficient $\mu(\boldsymbol{k})$ for $R e=1000$ and $\operatorname{Pr}=(1,1.5,4)$. For each wavenumber $\boldsymbol{k}$ with $-0.5 \leqslant k_{x} \leqslant 0.1$ and $0 \leqslant k_{y} \leqslant 1.2, \mu$ is computed for $R i \simeq R i_{c}(\operatorname{Re}, \operatorname{Pr}, \boldsymbol{k})$. For all such $\boldsymbol{k}$, the values of $\operatorname{Re}(\mu)$ for $\operatorname{Pr}=1$ are negative which means that the nonlinear terms have a stabilizing effect. For $\operatorname{Pr}=1.5, \operatorname{Re}(\mu)$ takes both signs (figure $5 b$ ). For $\operatorname{Pr}=4$ (figure $5 c$ ), the values of $\operatorname{Re}(\mu)$ become positive for most $\boldsymbol{k}$ and the nonlinear effect becomes almost everywhere destabilizing. Similar results are found at $R e=500$ (not shown). We conclude then that this stabilizing/destabilizing effect depends mainly on the Prandtl number.

\subsection{Effect of the Prandtl number on nonlinear feedback}

Table 2 presents the real part of the Landau coefficient $\mu$ and its various contributions for $\operatorname{Re}=1000, \operatorname{Pr}=0.25,0.7,1,4$. We observe again that $\operatorname{Re}\left(\mu_{b z}^{[0]}\right)$ is destabilizing and strongly enhanced as $P r$ increases, while the other contributions to $\mu^{[0]}$ are stabilizing and depend weakly on the Prandtl number. Combined with a small $\mu^{[2]}$, this results in a destabilizing net feedback $\operatorname{Re}(\mu)>0$ at $\operatorname{Pr}=4$. 

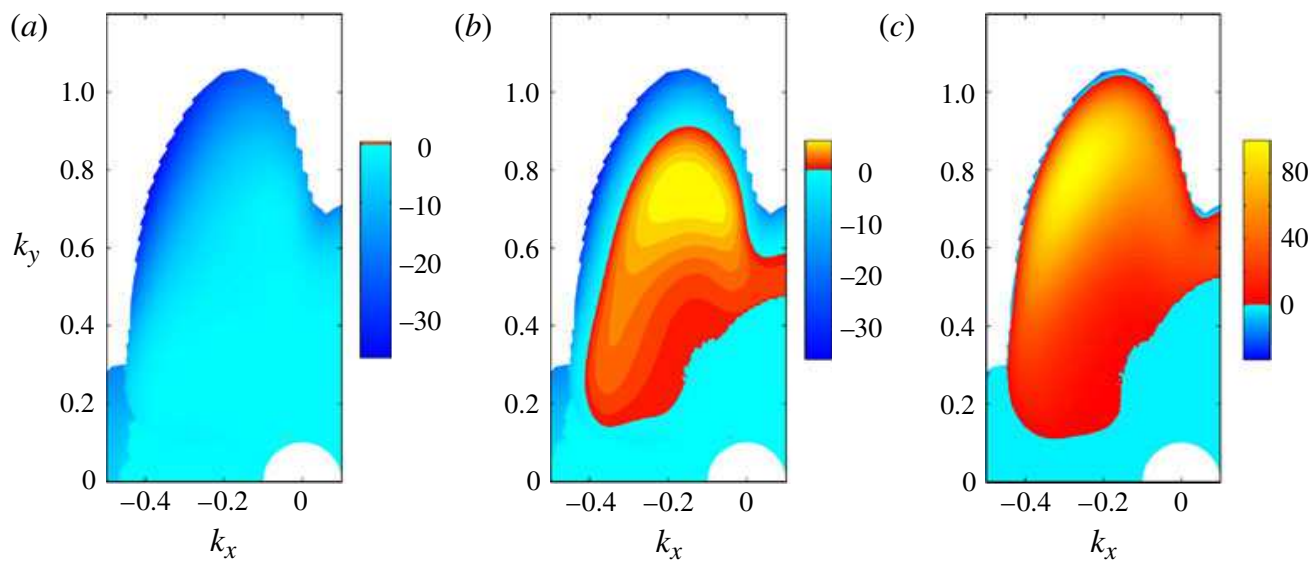

FIGURE 5. Real part of the Landau coefficient $\mu$ for $\operatorname{Re}=1000$ and for $\operatorname{Pr}=1(a), \operatorname{Pr}=1.5$

(b) and $\operatorname{Pr}=4(c)$. For each wave vector $\boldsymbol{k}, \mu$ is computed with $R i=\operatorname{Ri}(\operatorname{Re}, \operatorname{Pr}, \boldsymbol{k})$.

We present in figure $6(a)$ the modification of the mean shear $\mathrm{d} u_{2}^{[0]} / \mathrm{d} z$ and mean stratification $\mathrm{d} b_{2}^{[0]} / \mathrm{d} z$. Again, the interpretation of these profiles is that the mean velocity and buoyancy profiles change as the perturbation grows according to (3.2) with the Ekman basic flow replacing the $\mathrm{KH}$ profile. We see that $\mathrm{d} u_{2}^{[0]} / \mathrm{d} z$, like $\operatorname{Re}\left(\mu_{\boldsymbol{u}}^{[0]}\right)$, depends weakly on $\operatorname{Pr}$. Furthermore $\mathrm{d} u_{2}^{[0]} / \mathrm{d} z$ is mostly positive while $\mathrm{d} U_{E} / \mathrm{d} z$ is mostly negative (figure $4 b$ ), i.e. the mean shear weakens as the instability develops. On the other hand $\mathrm{d} b_{2}^{[0]} / \mathrm{d} z$ is also negative, i.e. the stratification also weakens as the instability develops. This effect is amplified when $\operatorname{Pr}=4$ due to the stronger buoyancy gradients that can form when the diffusivity is low. We plot next $\max \left(\left|\mathrm{d} u_{2}^{[0]} / \mathrm{d} z\right|\right)$ and $\max \left(\left|\mathrm{d} b_{2}^{[0]} / \mathrm{d} z\right|\right)$ (figure $6 b$ ) as a function of $\operatorname{Pr}$ at a fixed $R e$, and find that as in the case of KH flow, they scale roughly with $P r$.

We now turn to the scaling as a function of $R e$ at fixed $\operatorname{Pr}$. Figure 7(a) presents $\max \left(\left|\mathrm{d} u_{2}^{[0]} / \mathrm{d} z\right|\right)$ and $\max \left(\left|\mathrm{d} b_{2}^{[0]} / \mathrm{d} z\right|\right)$ for $\operatorname{Pr}=0.25,4$ and $500 \leqslant R e \leqslant 10000$. Note that we use $\operatorname{Pr}=0.25$ to make sure that the Landau coefficient does not change sign as $R e$ varies; $\max \left(\left|\mathrm{d} u_{2}^{[0]} / \mathrm{d} z\right|\right)$ (stars) and $\max \left(\left|\mathrm{d} b_{2}^{[0]} / \mathrm{d} z\right|\right)$ (circles) scale roughly with $R e$ as expected from the asymptotic theory of Churilov \& Shukhman (1987) and (3.3). To find if $\operatorname{Re}\left(\mu^{[0]}\right)$ and $\operatorname{Re}\left(\mu^{[2]}\right)$ also scale as $\operatorname{Re}$, we plot these two variables as a function of $\operatorname{Re}$ (figure $7 b$ ). Now $\operatorname{Re}\left(\mu^{[0]}\right)$ and $\operatorname{Re}\left(\mu^{[2]}\right)$ increase with $\operatorname{Re}$ for $\operatorname{Pr}=4$ but apparently not as fast as predicted by a linear scaling. Furthermore for $\operatorname{Pr}=0.25$, $\operatorname{Re}\left(\mu^{[0]}\right)$ and $\operatorname{Re}\left(\mu^{[2]}\right)$ actually decrease as $R e$ increases, quite the opposite of the expected behaviour. Therefore while the behaviour as a function of $\operatorname{Pr}$ is similar in the $\mathrm{KH}$ and Ekman flows, the scaling with $R e$ is not entirely the same, especially for low values of $\mathrm{Pr}$.

\subsection{Development and saturation of the inflection-point instability}

We now verify the conclusions of weakly nonlinear theory regarding the subcritical or supercritical character of the bifurcation at $R i=R i_{c}$ using numerically computed equilibrated flows, i.e. solutions of the fully nonlinear Boussinesq equations that are stationary in a moving reference frame. Those equilibrated flows have been computed by Mkhinini et al. (2013) and have a structure comparable to KH rolls. To identify the nature of the bifurcation, we compute in figure 8 the total energy $T E$ of the 

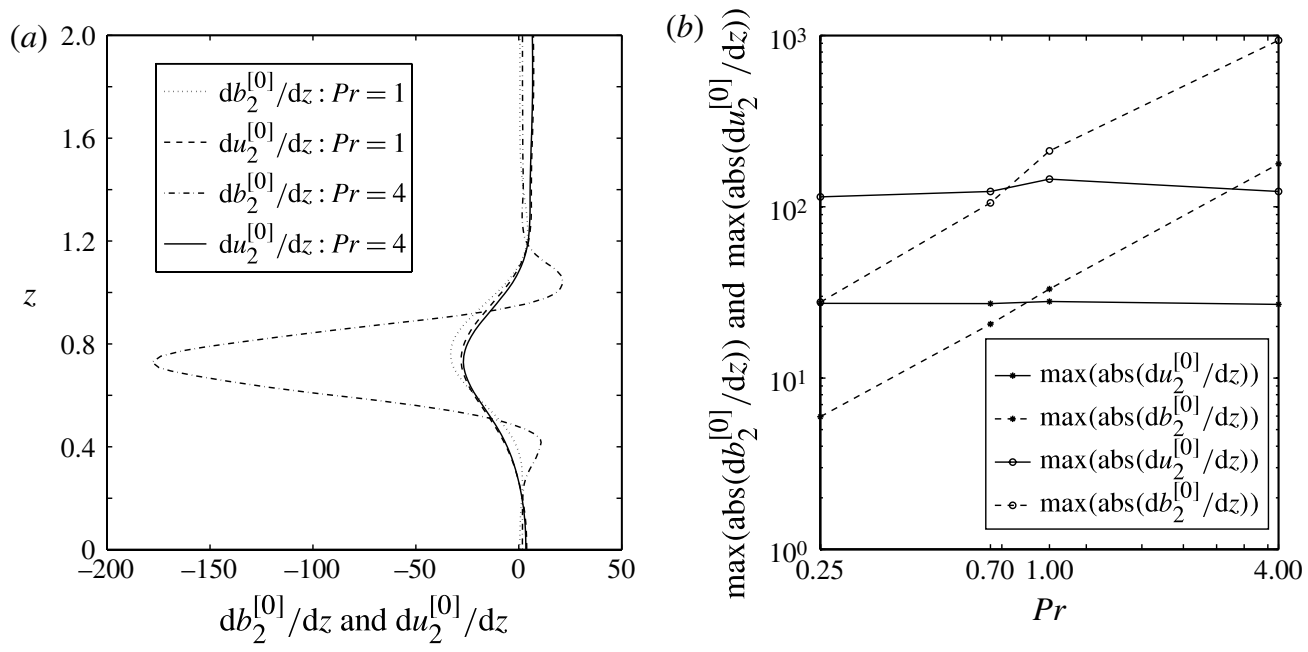

FIGURE 6. (a) Nonlinear modification of mean shear $\mathrm{d} u_{2}^{[0]} / \mathrm{d} z$ and mean stratification $\mathrm{d} b_{2}^{[0]} / \mathrm{d} z$ at $P r=1$ and $P r=4, R e=1000 .(b) \max \left(\left|\mathrm{d} u_{2}^{[0]} / \mathrm{d} z\right|\right)$ and $\max \left(\left|\mathrm{d} b_{2}^{[0]} / \mathrm{d} z\right|\right)$ as a function of $P r$ at $R e=1000$ (dots) and $R e=10000$ (circles) for Ekman flow.
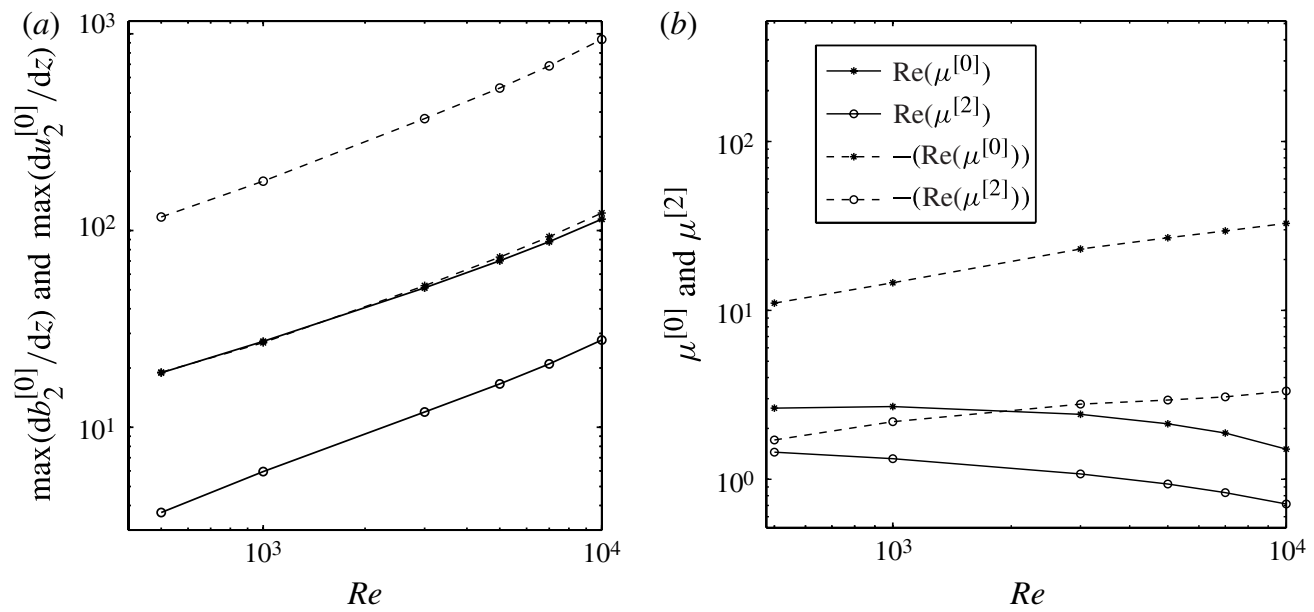

FIGURE 7. (a) $\max \left(\left|\mathrm{d} u_{2}^{[0]} / \mathrm{d} z\right|\right)$ (dots) and $\max \left(\left|\mathrm{d} b_{2}^{[0]} / \mathrm{d} z\right|\right)$ (circles) as a function of $R e$ for Ekman flow and $\operatorname{Pr}=0.25$ (solid) and $\operatorname{Pr}=4$ (dashed). (b) $\operatorname{Re}\left(\mu^{[0]}\right)$ and $\operatorname{Re}\left(\mu^{[2]}\right)$ as a function of $R e$ for Ekman flow. At $P r=4$ (dashed), $\mu^{[0]}$ and $\mu^{[2]}$ are positive while at $\operatorname{Pr}=0.25$ (solid), $\mu^{[0]}$ and $\mu^{[2]}$ are negative and we plot $-\mu^{[0]}$ and $-\mu^{[2]}$.

equilibrated rolls as a function of $R i$ for four pairs of parameters $(R e, P r)$. As expressed by (3.4), the amplitude of the nonlinearly equilibrated rolls vanishes as $R i$ approaches $R i_{c}$ if $\mu<0$. For $\operatorname{Pr}=1$, the kinetic and total energies vanish for $R i$ close to $R i_{c}$. On the other hand, for $\operatorname{Pr}=4$, the kinetic and total energies remain finite when $R i$ reaches $R i_{c}$. This shows that the bifurcation at $R i=R i_{c}$ for $P r=4$ is indeed of the subcritical type while the bifurcation is of the supercritical type at $\operatorname{Pr}=1$. 


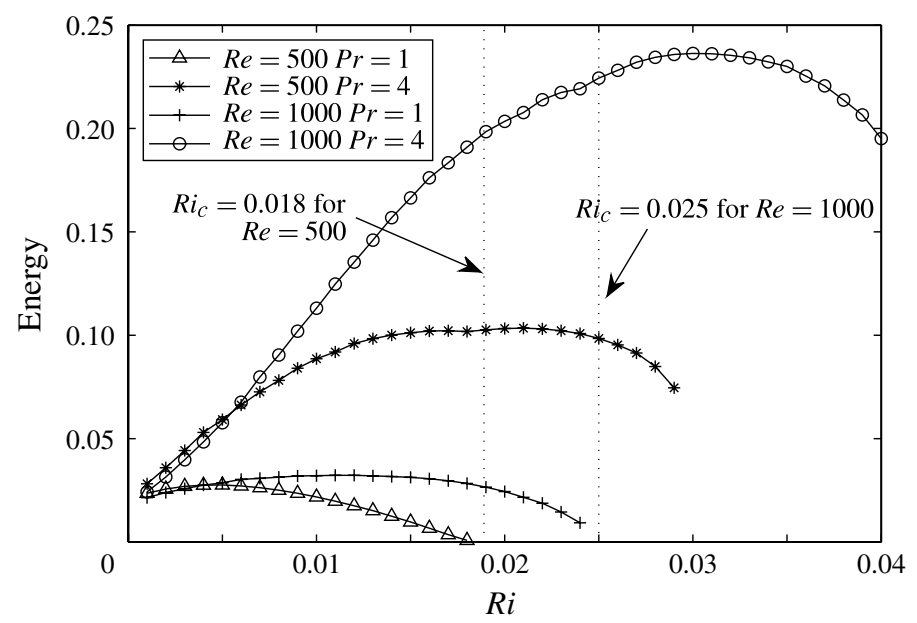

FIGURE 8. Total energy of equilibrated Ekman rolls as a function of $R i$ for several $(R e, P r)$. At $R e=500, R i_{c} \simeq 0.018$ and at $R e=1000, R i_{c} \simeq 0.025$.

\section{Discussion}

The present weakly nonlinear analysis confirms that the nature of the bifurcation of stratified Ekman flow near $R i=R i_{c}$ is dominantly controlled by the Prandtl number $P r$. Details of the asymptotic theory derived for the $\mathrm{KH}$ basic flow seem invalid for the Ekman basic flow. In particular, the scaling of the first Landau coefficient with $R e$ for $\operatorname{Pr}<1$ is not as predicted by Churilov \& Shukhman (1987). Nevertheless the dominant mechanism controlling the net nonlinear feedback remains that the mean flow adjusts so as to induce a viscous (respectively diffusive) flux of momentum (respectively buoyancy) that balances the vertical flux induced by the developing instability, leading to a Prandtl-number-independent weakening of the mean shear and a Prandtl-numberdependent weakening of the mean stratification. The competition between the former effect, which is stabilizing (as well as other contributions not analysed here in detail), and the latter effect, which is destabilizing, determines the supercritical or subcritical character of the bifurcation. Therefore the feedback mechanism identified by Brown et al. (1981) and Churilov \& Shukhman (1987) and its dependence on $\operatorname{Pr}$ can be expected to have a wide validity among stratified shear flows, even in the presence of veering and a solid boundary.

Typical values of $P r$ range from 0.7 for the diffusion of heat in air to $\sim 700$ for the diffusion of salt in water. We do not attempt to reach such high $\operatorname{Pr}$ because strong effects are already present at $P r=O(1)$. Reaching oceanic values would require either highly resolved numerical calculations or an asymptotic theory in the limit of high $P r$. This is beyond the scope of the present work but it is plausible that the nonlinear feedback we discuss remains valid at very high Prandtl numbers.

A consequence of this feedback mechanism is that finite-amplitude KH-like billows can occur even marginally below $R i_{c}$ at high $\operatorname{Pr}$, like in the ocean, while for low $\operatorname{Pr}$ like in the atmosphere a finite departure from $R i_{c}$ is needed to form billows. Furthermore finite-amplitude billows may develop even at $R i>R i_{c}$ at high $\operatorname{Pr}$ provided some mechanism generates perturbations with an initial amplitude large enough for the destabilizing nonlinear feedback to overcome the stabilizing linear feedback $\left(\sigma_{1}<0\right.$ if $R i>R i_{c}$ ). Efficient three-dimensional mixing in stratified shear flows results from 
a buoyant-convective secondary instability which depends on the ability of billows to form locally statically unstable regions (Peltier \& Caulfield 2003). $\operatorname{Pr}$ may therefore have a profound influence on such mixing, and it would be interesting and important to re-examine Prandtl number effects in stratified turbulence beyond the few existing studies (e.g. Klaassen \& Peltier 1985).

\section{Acknowledgements}

We are grateful to J.-M. Chomaz for fruitful discussions and suggestions, to C.-C. Caulfield for pointing us to previous work by Churilov \& Shukhman (1987), to R. Plougonven for carefully proofreading the manuscript, and to three anonymous referees who helped to improve the manuscript significantly. This work was supported by the INSU/LEFE program and by the Agence Nationale de la Recherche, contract ANR-09-JCJC-0108-01.

\section{Appendix A. Weakly nonlinear theory}

Several equivalent weakly nonlinear formalisms can be found in the litterature (Stuart 1960; Watson 1960; Reynolds \& Potter 1967; Herbert 1983; Fujimura 1989). Our formalism is along the lines of Herbert (1983). We let $\boldsymbol{U}=\boldsymbol{U}_{0}+\boldsymbol{u}$ with either $\boldsymbol{U}_{0}=\boldsymbol{U}_{E}$ or $\boldsymbol{U}_{0}=\boldsymbol{U}_{K H}$ and look for a solution of the Boussinesq equations (2.1)-(2.2) written formally as:

$$
\frac{\partial}{\partial t}\left[\begin{array}{l}
\boldsymbol{u} \\
b
\end{array}\right]-L \cdot\left[\begin{array}{l}
\boldsymbol{u} \\
b
\end{array}\right]+\boldsymbol{u} \cdot \nabla\left[\begin{array}{l}
\boldsymbol{u} \\
b
\end{array}\right]=0
$$

where $L$ represents the dynamics linearized about the basic flow:

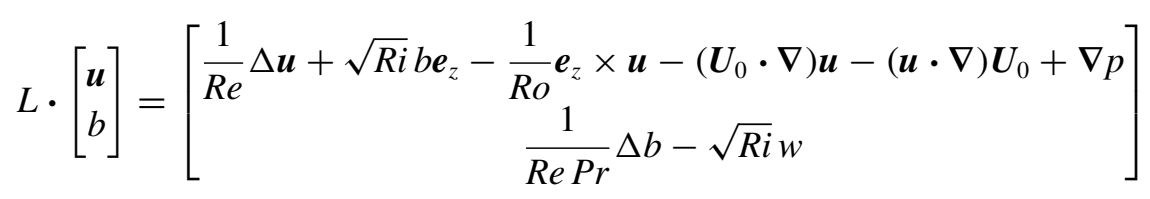

where $1 / R o=2 / R e$ for the Ekman flow and $1 / R o=0$ for the KH flow. In (A 2) we have explicitly written the contribution of the pressure gradient $\nabla p$ on the r.h.s. However in actual numerical computations this r.h.s. is projected onto a space of divergence-free velocity fields, and the pressure gradient does not contribute to the result of this projection. Therefore in what follows we do not include this pressure gradient term, although it is actually present and ensures the non-divergence of the flow. The deviation $\boldsymbol{u}, b$ from the laminar flow results from the nonlinear development of the primary instability with wave vector $\boldsymbol{k}$. We define linear operators acting on each Fourier mode separately:

$$
\begin{aligned}
L \cdot\left[\begin{array}{l}
E^{l} \boldsymbol{u}^{[l]}(z) \\
E^{l} b^{[l]}(z)
\end{array}\right] & =E^{l} L^{[l]} \cdot\left[\begin{array}{l}
\boldsymbol{u}^{[l]}(z) \\
b^{[l]}(z)
\end{array}\right], \\
\nabla\left[\begin{array}{l}
E^{l} \boldsymbol{u}^{[l]}(z) \\
E^{l} b^{[l]}(z)
\end{array}\right] & =E^{l} \nabla^{[l]}\left[\begin{array}{l}
\boldsymbol{u}^{[l]}(z) \\
b^{[l]}(z)
\end{array}\right], \\
\nabla^{[l]} & =\boldsymbol{e}_{x} \mathrm{i} k l+\boldsymbol{e}_{z} \frac{\partial}{\partial z}
\end{aligned}
$$


where $E=\mathrm{e}^{\mathrm{i} k \cdot x}, k=\|\boldsymbol{k}\|$ and $l$ indexes the Fourier modes. For the mean flow $l=0$, the expression for $L^{[0]}$ simplifies to:

$$
L^{[0]} \cdot\left[\begin{array}{l}
\boldsymbol{u} \\
b
\end{array}\right]=\left[\begin{array}{c}
\frac{1}{\operatorname{Re}} \frac{\mathrm{d}^{2} \boldsymbol{u}}{\mathrm{d} z^{2}}-\frac{1}{\operatorname{Ro}} \boldsymbol{e}_{z} \times \boldsymbol{u} \\
\frac{1}{\operatorname{Re} \operatorname{Pr}} \frac{\mathrm{d}^{2} b}{\mathrm{~d} z^{2}}
\end{array}\right]
$$

because $w=0, \partial / \partial x=0,\left(\boldsymbol{U}_{E} \cdot \nabla\right) \boldsymbol{u}=(\boldsymbol{u} \cdot \nabla) \boldsymbol{U}_{E}=0$ and the pressure gradient cancels the buoyancy force.

We expand $\boldsymbol{u}, b$ in powers of a complex amplitude $A$ assumed to be small:

$$
\boldsymbol{u}(\boldsymbol{x}, A)=\sum_{m=1}^{\infty} \boldsymbol{u}_{m}(\boldsymbol{x}, A, \bar{A}), \quad b(\boldsymbol{x}, A)=\sum_{m=1}^{\infty} b_{m}(\boldsymbol{x}, A, \bar{A})
$$

where $\boldsymbol{u}_{m}, b_{m}$ are polynomials of total degree $m$ in $A$ and its complex conjugate $\bar{A} ; \boldsymbol{u}_{m}$ and $b_{m}$ contain only even (respectively odd) Fourier modes when $m$ is even (respectively odd):

$$
\begin{aligned}
& {\left[\begin{array}{l}
\boldsymbol{u}_{1} \\
b_{1}
\end{array}\right]=A E\left[\begin{array}{l}
\boldsymbol{u}_{1}^{[1]} \\
b_{1}^{[1]}
\end{array}\right]+\bar{A} \bar{E}\left[\begin{array}{l}
\boldsymbol{u}_{1}^{[1]} \\
b_{1}^{[1]}
\end{array}\right],} \\
& {\left[\begin{array}{l}
\boldsymbol{u}_{2} \\
b_{2}
\end{array}\right]=A^{2} E^{2}\left[\begin{array}{c}
\boldsymbol{u}_{2}^{[2]} \\
b_{2}^{[2]}
\end{array}\right]+A \bar{A}\left[\begin{array}{c}
\boldsymbol{u}_{2}^{[0]} \\
b_{2}^{[0]}
\end{array}\right]+\bar{A}^{2} \bar{E}^{2}\left[\begin{array}{c}
\boldsymbol{u}_{2}^{[-2]} \\
b_{2}^{[-2]}
\end{array}\right], \ldots}
\end{aligned}
$$

Note that $\boldsymbol{u}_{m}^{[-l]}=\overline{\boldsymbol{u}_{m}^{[l]}}$ and $b_{m}^{[-l]}=\overline{b_{m}^{[l]}}$. Equation (A 7) satisfies (A 1) provided the amplitude evolves according to $\mathrm{d} A / \mathrm{d} t=A \sigma\left(|A|^{2}\right)=A\left(\sigma_{1}+\mu|A|^{2}+\cdots\right)$ where $\sigma_{1}$ and $\mu$ are to be obtained and the $\boldsymbol{u}_{m}^{[l]}, b_{m}^{[l]}$ satisfy relationships that we now detail up to order $m=3$. At first order in $A$ one finds the eigenproblem of the linear stability analysis

$$
L^{[1]} \cdot\left[\begin{array}{c}
\boldsymbol{u}_{1}^{[1]} \\
b_{1}^{[1]}
\end{array}\right]=\sigma_{1}\left[\begin{array}{c}
\boldsymbol{u}_{1}^{[1]} \\
b_{1}^{[1]}
\end{array}\right]
$$

We impose

$$
\left.\overline{\left\langle\boldsymbol{u}_{1}^{[1]}\right.} \cdot \boldsymbol{u}_{1}^{[1]}+\overline{b_{1}^{[1]}} b_{1}^{[1]}\right\rangle=1 \quad \text { where }\langle b\rangle=\frac{k}{2 \pi} \int_{[0,2 \pi / k] \times[0, \infty[} b \mathrm{~d} x \mathrm{~d} z,
$$

which allows $|A|^{2}$ to be interpreted, to leading order, as the total energy of the perturbation. In order to uniquely define the amplitude $A$, we introduce the adjoint eigenvector $\boldsymbol{v}, \beta$ subject to the normalization constraint $\left\langle\overline{\boldsymbol{v}} \cdot \boldsymbol{u}_{1}^{[1]}+\bar{\beta} b_{1}^{[1]}\right\rangle=1$ and define the amplitude as $A=\langle\bar{E}(\overline{\boldsymbol{v}} \cdot \boldsymbol{u}+\bar{\beta} b)\rangle$. The adjoint eigenproblem is posed at the discrete level: the adjoint eigenvector is defined as an eigenmode of the conjugatetranspose of the matrix representing operator $L^{[1]}$.

At second order in $A$ one finds $O\left(|A|^{2}\right)$ modifications of the flow, determined by the response of the mean flow and the $2 k$ mode to forcing by the nonlinear terms generated by the first harmonic:

$$
\left(L^{[0]}-\left(\sigma_{1}+\bar{\sigma}_{1}\right)\right)\left[\begin{array}{c}
\boldsymbol{u}_{2}^{[0]} \\
b_{2}^{[0]}
\end{array}\right]=\boldsymbol{u}_{1}^{[1]} \cdot \nabla^{[-1]}\left[\begin{array}{c}
\boldsymbol{u}_{1}^{[-1]} \\
b_{1}^{[-1]}
\end{array}\right]+\text { c.c. },
$$




$$
\left(L^{[2]}-2 \sigma_{1}\right)\left[\begin{array}{c}
\boldsymbol{u}_{2}^{[2]} \\
b_{2}^{[2]}
\end{array}\right]=\boldsymbol{u}_{1}^{[1]} \cdot \nabla^{[1]}\left[\begin{array}{c}
\boldsymbol{u}_{1}^{[1]} \\
b_{1}^{[1]}
\end{array}\right] \text {. }
$$

Considering the Fourier mode $l=1$ at third order in $A$, one finds how the amplitude must evolve consistently with $O\left(|A|^{3}\right)$ modifications of the flow:

$$
\begin{gathered}
\left(L^{[1]}-2 \sigma_{1}-\bar{\sigma}_{1}\right) \cdot\left[\begin{array}{c}
\boldsymbol{u}_{3}^{[1]} \\
b_{3}^{[1]}
\end{array}\right]+\mu\left[\begin{array}{c}
\boldsymbol{u}_{1}^{[1]} \\
b_{1}^{[1]}
\end{array}\right]=\left[\begin{array}{c}
\boldsymbol{u}_{S} \\
b_{S}
\end{array}\right]=\left[\begin{array}{c}
\boldsymbol{u}_{S}^{[0]} \\
b_{S}^{[0]}
\end{array}\right]+\left[\begin{array}{c}
\boldsymbol{u}_{S}^{[2]} \\
b_{S}^{[2]}
\end{array}\right], \\
{\left[\begin{array}{c}
\boldsymbol{u}_{S}^{[0]} \\
b_{S}^{[0]}
\end{array}\right]=\boldsymbol{u}_{2}^{[0]} \cdot \nabla^{[1]}\left[\begin{array}{c}
\boldsymbol{u}_{1}^{[1]} \\
b_{1}^{[1]}
\end{array}\right]+\boldsymbol{u}_{1}^{[1]} \cdot \nabla^{[0]}\left[\begin{array}{c}
\boldsymbol{u}_{2}^{[0]} \\
b_{2}^{[0]}
\end{array}\right],} \\
{\left[\begin{array}{c}
\boldsymbol{u}_{S}^{[2]} \\
b_{S}^{[2]}
\end{array}\right]=\boldsymbol{u}_{2}^{[2]} \cdot \nabla^{[-1]}\left[\begin{array}{c}
\boldsymbol{u}_{1}^{[-1]} \\
b_{1}^{[-1]}
\end{array}\right]+\boldsymbol{u}_{1}^{[-1]} \cdot \nabla^{[2]}\left[\begin{array}{c}
\boldsymbol{u}_{2}^{[2]} \\
b_{2}^{[2]}
\end{array}\right]}
\end{gathered}
$$

Now a consequence of the definition of $A$ and the adjoint eigenmode is that $\left\langle\overline{\boldsymbol{v}} \cdot \boldsymbol{u}_{3}^{[1]}+\bar{\beta} b_{3}^{[1]}\right\rangle=0$, which yields the compatibility condition determining $\mu$ and its decomposition:

$$
\mu=\left\langle\overline{\boldsymbol{v}} \cdot \boldsymbol{u}_{S}+\bar{\beta} b_{S}\right\rangle=\mu^{[0]}+\mu^{[2]}, \quad \mu^{[0]}=\mu_{u}^{[0]}+\mu_{b}^{[0]}, \quad \mu_{b}^{[0]}=\mu_{b x}^{[0]}+\mu_{b z}^{[0]}
$$

where

$$
\begin{aligned}
& \mu^{[2]}=\left\langle\overline{\boldsymbol{v}} \cdot \boldsymbol{u}_{S}^{[2]}+\bar{\beta} b_{S}^{[2]}\right\rangle=\left\langle\overline{\boldsymbol{v}} \cdot\left(\boldsymbol{u}_{2}^{[2]} \cdot \nabla^{[-1]} \boldsymbol{u}_{1}^{[-1]}+\boldsymbol{u}_{1}^{[-1]} \cdot \nabla^{[2]} \boldsymbol{u}_{2}^{[2]}\right)\right. \\
&\left.+\bar{\beta}\left(\boldsymbol{u}_{2}^{[2]} \cdot \nabla^{[-1]} b_{1}^{[-1]}+\boldsymbol{u}_{1}^{[-1]} \cdot \nabla^{[2]} b_{2}^{[2]}\right)\right\rangle, \\
& \mu_{\boldsymbol{u}}^{[0]}=\left\langle\overline{\boldsymbol{v}} \cdot\left(\boldsymbol{u}_{2}^{[0]} \cdot \nabla^{[1]} \boldsymbol{u}_{1}^{[1]}+\boldsymbol{u}_{1}^{[1]} \cdot \nabla^{[0]} \boldsymbol{u}_{2}^{[0]}\right)\right\rangle, \\
& \mu_{b x}^{[0]}=\left\langle\bar{\beta} \boldsymbol{u}_{2}^{[0]} \cdot \nabla^{[1]} b_{1}^{[1]}\right\rangle=\left\langle\mathrm{i} k \bar{\beta} u_{2}^{[0]} b_{1}^{[1]}\right\rangle, \\
& \mu_{b z}^{[0]}=\left\langle\bar{\beta} \boldsymbol{u}_{1}^{[1]} \cdot \nabla^{[0]} b_{2}^{[0]}\right\rangle=\left\langle\bar{\beta}_{1}^{[1]} \cdot \frac{\partial b_{2}^{[0]}}{\partial z}\right\rangle .
\end{aligned}
$$

\section{Appendix B. Numerical discretization}

In all circumstances we actually solve for the deviations $\boldsymbol{u}$ from the basic profile and $b$ from the linear background stratification. The vertical coordinate $z$ is mapped to $\eta \in[-1,1]$. The mapping is $\eta=\tanh z / z_{0}$ with $z_{0}=5.1$ in the $\mathrm{KH}$ case and $\eta=1-2 \mathrm{e}^{-z / z_{0}}$ with $z_{0}=4.1$ in the Ekman case. The interval $\eta \in[-1,1]$ is equally divided into $N_{z}$ elements, in each of which we define six Gauss-Legendre quadrature points. The free parameter $z_{0}$ controls the thickness of the well-resolved region close to $z=0$.

Discrete problems are obtained from the continuous problems by Galerkin projection onto finite-dimensional spaces for velocity and buoyancy satisfying horizontally periodic boundary conditions. For the vertical discretization we use $N_{z}+4$ cubic B-splines $S_{m}(\eta)$. Multiplying $S_{m}(\eta)$ by $f(\eta)=1-\eta$ in the Ekman case (respectively $f(\eta)=(1+\eta)(1-\eta)$ in the $\mathrm{KH}$ case) ensures exponential decay for $z \rightarrow \infty$ (respectively $z \rightarrow \pm \infty$ ). The velocity components $u=-\partial \psi / \partial z, w=\partial \psi / \partial x$ are defined 
by a stream function $\psi$ to ensure non-divergence:

$$
\begin{aligned}
& b(x, y, z)=\sum_{k=0}^{M_{x}} \sum_{m=m_{1}}^{N_{z}+4} \tilde{b}_{k m} \exp \left[\mathrm{i}\left(\frac{k x}{2 \pi L_{x}}\right)\right] f(\eta) S_{m}(\eta)+\text { c.c. }, \\
& \psi(x, y, z)=\sum_{k=1}^{M_{x}} \sum_{m=m_{2}}^{N_{z}+4} \tilde{\psi}_{k m} \exp \left[\mathrm{i}\left(\frac{k x}{2 \pi L_{x}}\right)\right] f(\eta) S_{m}(\eta)+\text { c.c. }, \\
& v(x, y, z)=\sum_{k=0}^{M_{x}} \sum_{m=m_{1}}^{N_{z}+4} \tilde{v}_{k m} \exp \left[\mathrm{i}\left(\frac{k x}{2 \pi L_{x}}\right)\right] f(\eta) S_{m}(\eta)+\text { c.c. }
\end{aligned}
$$

where $\boldsymbol{u}(x, y, z)=U(z) \boldsymbol{e}_{x}+u(z) \boldsymbol{e}_{x}+v(z) \boldsymbol{e}_{y}+w(z) \boldsymbol{e}_{z},\left(m_{1}, m_{2}\right)=(0,0)$ in the KH case and $\left(m_{1}, m_{2}\right)=(1,2)$ in the Ekman case. The B-splines satisfy $\mathrm{d} S_{m} /\left.\mathrm{d} \eta\right|_{\eta=-1}=0, m \geqslant 1$ and $\mathrm{d}^{2} S_{m} /\left.\mathrm{d} \eta^{2}\right|_{\eta=-1}=0, m \geqslant 2$ so that in the Ekman case the boundary conditions at $z=0$ are enforced by excluding the first or first two B-splines from the expansions. The expansion of the stream function $\psi$ does not include the $x$-mean profile $U(z)$ (corresponding to $k=0$ ) because this would imply $\int U(z) \mathrm{d} z=0$. Instead the mean profile $U(z)$ is expanded as a linear combination of $f(\eta) S_{m}(\eta), m \geqslant m_{1}$. The resulting fourth-order method is the same as the one used by Mkhinini et al. (2013), itself a modification of the spectrally accurate spatial discretization described in more detail in Spalart (1989) and used in earlier studies of the Ekman boundary layer (Coleman, Ferziger \& Spalart 1990; Foster 1996; Dubos et al. 2008). A vertical resolution of $N_{z}=60$ is sufficient at $\operatorname{Re}=1000, \operatorname{Pr}=4$ but $N_{z}=200$ was used to reach $\operatorname{Re}=10000$. For the weakly nonlinear computations the expansions (B 1)-(B 3) are truncated to $M_{x}=2$. Fully nonlinear simulations use $M_{x}=21$ with 64 quadrature points in the $x$ direction and Runge-Kutta time stepping.

\section{REFERENCES}

BROWN, R. A. 1972 On the inflection point instability of a stratified Ekman boundary layer. J. Atmos. Sci 29 (5), 850-859.

Brown, S. N., Rosen, A. S. \& Maslowe, S. A. 1981 The evolution of a quasi-steady critical layer in a stratified viscous shear layer. Proc. R. Soc. Lond. A 375 (1761), 271-293.

Churilov, S. M. \& Shukhman, I. G. 1987 Nonlinear stability of a stratified shear flow: a viscous critical layer. J. Fluid Mech. 180, 1-20.

Coleman, G. N., Ferziger, J. H. \& Spalart, P. R. 1990 A numerical study of the turbulent Ekman layer. J. Fluid Mech. 213, 313-348.

Coleman, G. N., Ferziger, J. H. \& Spalart, P. R. 1992 Direct simulation of the stably stratified turbulent Ekman layer. J. Fluid Mech. 244, 677-712.

Cortesi, A. B., Yadigaroglu, G. \& BanerJee, S. 1998 Numerical investigation of the formation of three-dimensional structures in stably-stratified mixing layers. Phys. Fluids 10 (6), 1449-1473.

DAVies, C. \& CARPenter, P. W. 2003 Global behaviour corresponding to the absolute instability of the rotating-disc boundary layer. J. Fluid Mech. 486, 287-329.

Dubos, T., Barthlott, C. \& Drobinski, P. 2008 Emergence and secondary instability of Ekman layer rolls. J. Atmos. Sci 65 (7), 2326-2342.

EkMAN, V. W. 1905 On the influence of the earth's rotation on ocean currents. Ark. Mat. Astron. Fys. 2 (11), 1-54.

Foster, R. C. 1996 An analytic model for planetary boundary layer roll vortices. PhD thesis, University of Washington. 
FUJIMURA, K. 1989 The equivalence between two perturbation methods in weakly nonlinear stability theory for parallel shear flows. Proc. R. Soc. Lond. A 424 (1867), 373-392.

Haeusser, T. M. \& Leibovich, S. 2003 Pattern formation in the marginally unstable Ekman layer. J. Fluid Mech. 479, 125-144.

Herbert, T. 1983 On perturbation methods in nonlinear stability theory. J. Fluid Mech. 126, $167-186$.

Howard, L. N. 1961 Note on a paper of John W. Miles. J. Fluid Mech. 10 (4), 509-512.

Iooss, G., Nielsen, H. \& True, H. 1978 Bifurcation of the stationary Ekman flow into a stable periodic flow. Arch. Rat. Mech. 68 (3), 227-256.

Klafissen, G. P. \& Peltier, W. R. 1985 The effect of Prandtl number on the evolution and stability of Kelvin-Helmholtz billows. Geophys. Astrophys. Fluid Dyn. 32 (1), 23-60.

LiLly, D. K. 1966 On the instability of Ekman boundary flow. J. Atmos. Sci. 23 (481-494).

Lingwood, R. J. 1997 Absolute instability of the Ekman layer and related rotating flows. J. Fluid Mech. 331, 405-428.

Lott, F. \& Teitelbaum, H. 1992 Nonlinear dissipative critical level interaction in a stratified shear flow: instabilities and gravity waves. Geophys. Astrophys. Fluid Dyn. 66 (1-4), 133-167.

Miles, J. W. 1961 On the stability of heterogeneous shear flows. J. Fluid Mech. 10 (4), 496-508.

Mkhinini, N., Dubos, T. \& Drobinski, P. 2013 Secondary instability of stratified Ekman layer. J. Fluid Mech. 728, 29-57.

Peltier, W. R. \& Caulfield, C. P. 2003 Mixing efficiency in stratified shear flows. Annu. Rev. Fluid Mech. 35, 135-167.

PIER, B. 2003 Finite-amplitude crossflow vortices, secondary instability and transition in the rotating-disk boundary layer. J. Fluid Mech. 487, 315-343.

Reynolds, W. C. \& Potter, M. C. 1967 Finite-amplitude instability of parallel shear flows. J. Fluid Mech. 27 (3), 465-492.

SPALART, P. R. 1989 Theoretical and numerical study of a three-dimensional turbulent boundary layer. J. Fluid Mech. 205, 319-340.

Staquet, C. 2009 Mixing in a stably stratified shear layer: two- and three-dimensional numerical experiments. Fluid. Dyn. Res., 27, 367.

STUART, J. T. 1960 On the nonlinear mechanics of wave disturbances in stable and unstable parallel flows. Part 1. The basic behaviour in plane Poiseuille flow. J. Fluid Mech. 9 (3), 353-370.

Viaud, B., Serre, E. \& Chomaz, J.-M. 2008 The elephant mode between two rotating disks. J. Fluid Mech. 598, 451-464.

WATSON, J. 1960 On the nonlinear mechanics of wave disturbances in stable and unstable parallel flows. Part 2. The development of a solution for plane Poiseuille flow and for plane Couette flow. J. Fluid Mech. 9 (3), 371-389. 